\title{
AN INTRODUCTORY REVIEW OF OUR PRESENT UNDERSTANDING OF THE STRUCTURE AND COMPOSITION OF URANUS' ATMOSPHERE
}

\author{
Michael J. S. Belton \\ Kitt Peak National Observatory $\uparrow$ \\ Tucson, Arizona 85726
}

Measurements of the gross properties of Uranus are enough to show that it is a planet with quite unique characteristics. The best estimates of its radius $(24900 \mathrm{~km}$; Danielson, Tomasko and Savage, 1972) and of its mass (14.54 Earth masses; Klepczynski, Seidelmann and Duncombe, 1970) imply an interior structure and overall composition that set it clearly apart from Jupiter and Saturn (Hubbard and MacFarlane, 1980).

The best estimates of the maximum outflow of internally generated heat (< 250 ergs $\mathrm{cm}^{-2} \mathrm{sec}^{-1}$; cf. Wallace, 1980) and its peculiar axial orientation (98 obliquity; Dunham, 1971) set it apart from its otherwise superficially similar sister planet, Neptune, when considering problems of atmospheric structure.

In this review, I will attempt to summarize the enormous progress that I believe has been made during the past decade (the average number of research papers regarding Uranus' atmosphere has increased from an average of one per year in the late 1960's to an average of $\sim 13$ papers per year in 1980) in understanding the planet's spectrum in terms of the probable chemical and physical nature of its atmosphere.

Essentially, all the work which has been done so far is exploratory in nature and I cannot report that a concensus on the/mean structure and composition of the planet's atmosphere has yet been achieved; there are, moreover, several important areas that need to be further explored before such a concensus can be expected. I am thinking particularly of questions concerning the time dependence of atmospheric structure which might occur as a result of the extreme seasonal characteristics of Uranus. On Uranus, unlike other planets, the polar regions receive an average of $\pi / 2$ more insolation than the equatorial regions. It is, nevertheless, possible to identify the general domain of the mean structure and composition and, perhaps more significantly, identify some of the critical observations on which future advances may depend.

toperated by the Association of Universities for Research in Astronomy, Inc., under contract with the National Science Foundation. 
I begin by presenting a thumbnail sketch of our current view of thi atmosphere in the context of a comparison with Neptune's atmosphere which, in the past, has often been assumed to have similar properties. This similarity of the two planets is now, I believe, very much in question.

\section{A Thumbnail Sketch}

So far as we know, the primary atmospheric constituents of the visible atmosphere ( $\mathrm{H}_{2}$ and $\mathrm{He}$ ) represent only 11 percent of the planet's mass (Hubbard and MacFarlane, 1980) and, if an interface between this atmos. phere and the ice-rock mixture that makes up the bulk of the planet, in fac exists, is about $7800 \mathrm{~km}$ deep with a base pressure near 221,000 bars at a temperature of $2500^{\circ} \mathrm{K}$.

of the sensible atmosphere itself, our observations have probed, albeit crudely in many cases, the region from $\sim 10 \mu$ bars to near the 100 bar pressure level. Above $10 \mu$ bars, we are in the realm of aeronomical conjecture which I shall not review although some exploratory work has been published by McElroy (1973) and by Capone et al (1977). 100 bars is about the deepest level that it has been possible to probe with microwave radiometry (21 cm observations; Briggs, 1973).

The Uranian stratosphere ( $10 \mu$ bar - 0.05 bar) is surprisingly cold $\left(\sim 95^{\circ} \mathrm{K}\right)$ while that of Neptune has a temperature $\left(\sim 140^{\circ} \mathrm{K}\right)$ and emission properties that are more in concert with our experience at Jupiter and Saturn. This may imply small but significantly different tropopausal struc tures on the two planets, in spite of their almost identical effective temperatures, since it is the tropopause temperature which probably control the stratosphere methane content and, thereby, its thermal balance with solar insolation. The meaning of "significant" can be inferred by noting that only a $3^{\circ} \mathrm{K}$ change in the assumed effective temperature could lead to a factor of two in the inferred stratospheric temperature (wallace, 1975) on account of the sensitivity of the vapor pressure of $\mathrm{CH}_{4}$ on temperature. Evidence that such differences do exist in the tropopausal region ( $50 \mathrm{~m}-$ bar level) is evidenced by the presence of cloud and "weather" at this level on Neptune (Joyce, Pilcher, Cruikshank and Morrison, 1977) but not on Uranus.

Between 0.1 and 10.0 bar, where the bulk atmosphere is in most effective radiative contact with space, the controlling thermal opacity (collisionally induced translational transitions in $\mathrm{H}_{2}-\mathrm{He}$ ) and the "effec tive" temperature are essentially identical on the two planets. As a consequence, the structures in this region should be very similar; a fact to which the visible and near infrared spectrum atests. In this region, the scale height is about $40 \mathrm{~km}$ and the lapse is near $0.8^{\circ} \mathrm{K} \mathrm{km}^{-1}$; methane condenses in this region, solar heating has its peak value, and there are 
indications of a system of layered clouds.

At pressures greater than a few tens of bars ( 230 bar; cf. wallace, 1980) the thermal structure is very sensitive to the actual cloud structures that are present (they control the deposition of solar heat) and the internal heat flux. Since it seems that Neptune's internal heat flow ( 300 ergs $\mathrm{cm}^{-2} \mathrm{sec}^{-1}$ ) is at least two times that of Uranus (and perhaps much more) then it seems to me quite possible that the two planets might exhibit different structures at these levels. On the other hand, the microwave spectra $(1-21 \mathrm{~cm})$ which are thought to probe these regions on both planets are very similar (cf., Morrison and Cruikshank, 1973) and, even though the shape of either spectrum has yet to have a satisfactory explanation (Gulkis, Janssen, and Olsen, 1978), this probably means similar atmosphere structures at these depths (10 - 100 bar).

Before moving into a more detailed discussion and since this paper is in the nature of an introductory review, I would now briefly like to note a few of the speculations that have crept into the research literature and which are now known to be definitely incorrect. This should be helpful to new readers of the literature and also helps to salve my own conscience, since I helped perpetuate some of them. The idea that extended wings in collisionally induced dipole transitions in $\mathrm{H}_{2}$ provide substantial opacity throughout the near infrared (Belton, McElroy, and Price, 1971) is no longer necessary or useful; the idea that simultaneous transitions in a $\mathrm{CH}_{4}-\mathrm{H}_{2}$ mixture have a role in producing the near infrared spectrum (Daniêlson, 1974) has been disapproved by new and extensive laboratory spectroscopy by Fink, Benner and Dick (1977) and by Giver (1978); the idea that no detectable aerosols exist in the Uranus atmosphere (Belton et al, 1971) has been disproved by proper treatment of radiative transport in the $\mathrm{H}_{2}$ quadropole lines and observations of the $(3-0) \mathrm{S}(0)$ collisionally induced feature of $\mathrm{H}_{2}$ at $8250 \AA$ (Trafton, 1976; Belton and Spinrad, 1973); confusion in the literature on the value of the transition movements of lines in the $(4-0)$ band of $\mathrm{H}_{2}$ (cf., Wallace, 1980) has now been cleared up with further laboratory work by Brault and Smith (1980) and the measured values are close to the theoretically computed value (Dalgarno and Allison, 1969). Thus, Trafton's (1976) work in interpreting the $\mathrm{H}_{2}$ spectrum of Uranus remains valid; finally, speculations that have surfaced from time to time regarding the presence, in large quantities, of a spectroscopically inert gas other than He (eg., from the studies of sharp $\mathrm{CH}_{4}$ lines near $6819 \AA$ ) all appear now to be without any real basis.

\section{A Stroll through Uranus' Spectrum}

As an introduction to a discussion on structure, let me now briefly review the character of the Uranian spectrum from whence the major part of our knowledge comes. This emphasis, of course, is not meant to 
deny the exceedingly important contributions from photometry (albedo; Younkin, 1970); from occultation measurements (stratospheric temperatures; Dunham, Elliot and Gierasch, 1980); from polarimetry (eg., Michalsky and Stokes, 1977; presence of upper atmospheric aerosols); from imagery (dimensions; limb brightening/darkening; Danielson et al, 1972; Sinton, 1972); and from center-to-limb observations (atmospheric inhomogeniety; $\mathrm{CH}_{4}$ saturation; Price and Franz, 1979; Pilcher et al., 1979).

In the ultraviolet, the shortest wavelengths at which, to my knowledge, the planet has been observed is $1800 \AA$ by the ANS satellite (Savage, Cochran and Wesselius, 1980). The planetary spectrum between $1800 \AA-4200 \AA$, which is superposed on the reflected solar spectrum, is dominated by the effects of rotational Raman transitions in $\mathrm{H}_{3}$ (Belton, Wallace and Price, 1973) and a diffuse absorption continuum whose origin is, at present, undetermined. At one time, I considered that observations of the Raman effect would impose significant boundary conditions on the distribution and amount of aerosols in the visible atmosphere, but this has not turned out to be the case and almost any of the many models considered so far for the Uranus atmosphere will satisfy the observations. The Raman transitions do, however, make a significant statement about the ortho/para ratio of $\mathrm{H}_{2}$ in the atmosphere.

The underlying absorption may well be the same "Axel" dust that exists in the upper atmospheres of Jupiter and Saturn - a photochemical detrius - but this is ad hoc and some of the absorption may be associated with one of the atmospheric cloud layers.

From $4200 \AA$ out to nearly $5 \mu$, the spectrum is dominated by a complex progression of $\mathrm{CH}_{4}$ bands. Recent and extensive laboratory work by Lutz, Owen, and Cess (1976), by Fink, Benner, and Dick (1977), by Giver (1978), and by Silvaggio (1977); and some measurements at low temperatures (in the stronger bands) have enabled the first quantitative attempts of explaining the planetary spectrum in terms of the complex coupling between the abundance of $\mathrm{CH}_{4}$ and atmospheric structure (Wallace, 1980). The socalled "blue" bands ${ }^{4}$ near $4800 \AA$ are found to place the most significant constraints on the deep $\mathrm{CH}_{4} / \mathrm{H}_{2}$ mixing ratio since they are weak enough for the radiation field to probe below the level of $\mathrm{CH}$ saturation; the stronger bands near $1 \mu$ put strong constraints on the abundance of upper atmospheric aerosols and the $\mathrm{He} / \mathrm{H} 2$ ratio but not on the abundance of $\mathrm{CH}_{4}$; finally, but in a less constructive way, the weak interband absorption in $\mathrm{CH}_{4}$ severely limits the interpretation of the $(3-0)$ collisionally induced dipole absorption of $\mathrm{H}$ near $8250 \AA$ which is very important for constraining cloud structure (Traftón, 1976) and measurements in the laboratory at low temperature are urgently needed. 
Superposed in the $\mathrm{CH}_{4}$ spectrum in this region is the relatively sparce spectrum of $\mathrm{H}_{2}$ and $\mathrm{HD}$. HD has, I think, been detected (Trafton and Ramsey, 1980; Trauger et al, 1977) in the $(5-0)$ band and the mixing ratio with $\mathrm{H}_{2}$ is close to $4 \cdot 8 \times 10^{-5}$, similar to Jupiter and Saturn. This result evidently must be taken into consideration in condensation scenarios for the planet (Hubbard and MacFarlane, 1981) for enhancements of a factor of two or more in Uranus and Neptune are expected. Perhaps on Uranus, D is still "trapped" in a partially solid ice ball? Observations of HD in Neptune are obviously of great interest.

The observed $\mathrm{H}_{2}$ spectrum consists of six lines of the quadrupole spectrum $(5-0,4-0$, and $3-0$ bands) and one line of the pressure induced dipole spectrum $(S(0)$ in the $3-0$ band). The relative strengths of lines from odd and even rotational levels indicate that the $\mathrm{H}_{2}$ rotational populations are close to thermal equilibrium for the temperatures in Uranus' upper atmosphere. (This is also indicated by the Raman spectrum.)

The $\mathrm{H}_{2}$ spectrum has turned out to be the crucial element in understanding cloud structure in the visible part of the atmosphere (cf., Trafton, 1976). The (3 - 0) quadrupole and dipole lines require a base cloud at a depth of $\sim 500 \mathrm{~km}$ - amagats of $\mathrm{H}_{2}$ ( $\sim 5$ bar level); while the $(4-0)$ lines require, in addition, a thin, ${ }^{2}$ tenuous, haze high in the atmosphere ( $\sim 0.3$ at $6400 \AA)$. The haze is ineffective if placed low in the atmosphere while its highest possible altitude is restricted by the observed albedo in the bottom of strong $\mathrm{CH}_{4}$ bands. The 1 bar level appears to be appropriate for this haze. Uncertainties in the observed strength of the (4 - 0) lines (Smith, Macy, and Pilcher, 1980) lead to considerable uncertainties in the properties of this elevated haze layer, although Wallace (1980) finds it an essential component in balancing the predicted strength of the $\mathrm{CH}_{4}$ "blue" band spectrum with the $(3-0)$ dipole feature.

It might be expected that at $5 \mu$, both Uranus and Neptune should easily be detected in reflected sunlight for molecular and aerosol scattering represents the only known and unexpected opacity. The planet has been detected (Gillett and Rieke, 1977; Macy, Sinton, and Beichman, 1980), but with the very low albedo of $\sim 0.01$. Macy et al suspect $\mathrm{CH}_{3} \mathrm{D}$ and $\mathrm{PH}_{3}$ as being the source of this absorption and spectra in this region are urgently needed.

Beyond $5 \mu$ is the undisputed domain of thermal radiation and the observed spectrum is dominated by collisionally induced rotational and translational transitions in $\mathrm{H}_{2}$. Stratospheric emissions of stratospheric hydrocarbons at 7.8 and $12 \mu$ have not yet been observed on Uranus and new efforts are clearly needed since I am not aware of any work since that of Gillett and Rieke (1977) or Macy and Sinton (1977). The stratosphere is 
not a total unknown as a result of successful observations of a grazing occultation of SAO 158687 by the planet from the Kuiper Airborne Observatory in 1977 (Dunham, Eliott and Gierasch, 1980) for a reasonable mixture of $\mathrm{H}$ and these observations indicate a temperature of $\sim 95^{\circ} \mathrm{K}$ at the $10 \mu$ bar level considerably cooler than is the case for the other giant planets.

Between 10 and $30 \mu$ where opacity due to the $S(0)$ and $S(1)$ lines in $\mathrm{H}_{2}$ (collisionally induced dipole rotational transitions) should allow probing of the upper troposphere and possibly the lower stratosphere, the observations are broad band radiometric measurements and are too crude to establish whether an inversion definitely exists in the 0.01 bar region (cf., Courtin, Goutier, and Lacombe, 1978), but they suffice to demonstrate the reasonableness of radiative-convective model atmospheres (Wallace, 1980).

From $30 \mu$ to a few $\mathrm{mm}$, the spectrum provides what is perhaps its most important constraint on atmospheric structure. This is because the bulk of the planetary thermal radiation emerges from the atmosphere in this spectral region where the opacity is controlled by collisionally induced dipole absorption between translational states of $\mathrm{H}_{2}$ and $\mathrm{He}$. Deep in the atmosphere, $\mathrm{CH}_{4}$ also plays an important role in the magnitude of this opacity (Wallace, 1980) but in the region of the visible atmosphere, the abundance of $\mathrm{CH}_{4}$ is depleted by condensation and is, therefore, not a factor in determining structure (Trafton, 1972). The various measurements show that the effective temperature is within 2 degrees of $58^{\circ} \mathrm{K}$ (Courtin et al, 1978; Stier et al., 1978) which restricts the internal outflow of internal heat to less than 250 ergs $\mathrm{cm}^{-2} \mathrm{sec}^{-1}$. Theoretical estimates of the internal heat flow range from $100 \mathrm{erg} \mathrm{cm} \mathrm{cm}^{-2} \mathrm{sec}^{-1}$ to 10 ergs $\mathrm{cm}^{-2} \mathrm{sec}^{-1}$ (Danielson, 1975) and could possibly be lower.

$\mathrm{H}_{2}$ - He opacity controls the spectrum to about $1 \mathrm{~cm}$ wavelength at which point the spectrum becomes exceedingly interesting and problematical. In the region between $1-21 \mathrm{~cm}$, the spectrum was anticipated to show brightness temperatures near $150^{\circ} \mathrm{K}$ and a shape that reflected the powerful absorption associated with inversion transitions in $\mathrm{NH}_{3}$. A "flat" spectrum was expected because of the high pressure ( $10^{3} \mathrm{bar}$ ) at which the spectrum would be formed and as a result of the vapor pressure characteristics of $\mathrm{NH}_{3}$ which cause a rapid increase in opacity over a limited vertical extent in the atmosphere. It was, therefore, a great surprise (albeit delayed) when Gulkis (1975) pointed out the fact that the spectrum is unexpectedly hot at $2-10 \mathrm{~cm}$; a surprise that was compounded when Klein and Turanago (1978) found the micro wave spectrum to be (at least at the present time) changing with time. Even at the present time, the shape of the Uranus microwave spectrum from $2-21 \mathrm{~cm}$, which is shared by Neptune, has defied adequate explanation. 


\section{Structure and Composition}

\section{a. General Considerations}

In a deep, cold, atmosphere like that which exists on Uranus, both the scattering of radiation and compositional inhomogeniety are of first order importance in the process of spectral line and band formation. As a result, we have learned by experience that the compositional problem and the structural problem are inextricably mixed. Most of the work to date has been of an exploratory nature; attempts to understand in the widest sense the complex interactions between assumed composition, cloud structure, and thermal structure. With the work of Danielson and his associates (1975, 1977), Trafton (1976), Courtin, Gautier and Lacombe (1978), and Wallace (1980), we have, I believe, arrived in the correct domain of structure and composition but, as yet, still lack a firm quantitative understanding.

Schematically, there are two approaches available for probing the atmospheric structure: "inverting" the observed spectral data on the basis of a set of rather restrictive and ad hoc assumptions (composition, behavior of the structure near upper and lower boundaries of the region probed) and the equation of radiative transfer; and, secondly, constructing sets of highly parameterized but physically consistent and reasonable radiative-convective models and comparing the predicted spectrum with observations. The inversion method has been applied by courtin et al (1978) and serves to give a crude idea of the general domain of possible models. The inversion method is, I believe, unsuitable for more refined work at the present time because of the very poor quality of the $10-300$ $\mu$ data that must be used (but see G. Orton's contribution to this colloquium).

The "modelling" method is technically more stable and forgiving and, in my view, is much more informative as it clearly exposes the influences of the physical processes that are at work and also help to identify whatever shortcomings exist in the observational data. This is the technique used by Danielson and his associates $(1975,1977)$, by Trafton (1976), by Macy (1979), and most recently by wallace (1980). The remainder of this review is concerned with this latter approach.

In creating a model, the following steps are normally employed:

(A) Assumptions regarding composition and internal heat flow

(B) First guess at a thermal. structure (i.e., the dependence of 
temperature on pressure throughout the atmosphere)

(C) Imposition of cloud characteristics

(D) Calculation of solar heat deposition

(E) Correction of initial thermal structure by iteration on step (B) to achieve a constant net energy flux condition throughout the atmosphere.

In step (A), the molecules and atoms we expect to be important for the visible atmosphere are a subset of those seen on Jupiter, but primarily $\mathrm{H}_{2}, \mathrm{He}, \mathrm{CH}_{4}$ and $\mathrm{NH}_{3}$. Prinn and Lewis (1973) have noted that $\mathrm{H}_{2} \mathrm{~S}$ may be an important molecule in the lower regions of the visible atmosphere and that it could be substantially enhanced (relative to $\mathrm{NH}_{3}$ ) over solar proportions as a result of some possible scenarios for the ${ }^{3}$ planet's origin. An $\mathrm{He} / \mathrm{H}_{2}$ ratio near 0.18 (e.g. Wallace, 1980) is used in al] the modern models, and only the (very significant) effects of $\mathrm{CH}$ condensation need be considered in structural models of the visible atmosphere. The $\mathrm{CH}_{4} / \mathrm{H}_{2}$ mixing ratio is taken as a free parameter in most models and the most satisfactory models have it enhanced some 40 times over the solar value $\left(\sim 7 \times 10^{-4}\right) . \mathrm{NH}_{3}$ and $\mathrm{H}_{2} \mathrm{~S}$ may have significant effects on the microwave spectrum but are apparently unimportant in structure calculations. Hunten (1981) has considered the effects of $\mathrm{H}_{2} \mathrm{O}$ on the structure but these are all confined to depths below 100 bars which are currently outside the limits of the observable atmosphere.

Limits exist for line of sight abundances of $\mathrm{NH}_{3}$ and $\mathrm{H}_{2} \mathrm{~S}$ (Fink and Larson, 1979) but a proper interpretation of these 3 limits in terms of deep mixing ratios has not been attempted and would be, I think, an exceedingly difficult task.

In step (C), two approaches have been used. In the first, a minimum of clouds and hazes are placed in the model atmosphere where needed in order to get a satisfactory rendition of the observed spectrum. Wallace (1980) who employs variations on Trafton's (1976) cloud models uses this approach as does Macy (1979). When a satisfactory physical structure is achieved, the models are then examined to see if the assumed cloud layers can be identified with potential condensates in the atmosphere. In these models, $\mathrm{CH}$ forms a high haze near $\mathrm{l}$ bar with a thick cloud base of $\mathrm{H}_{2} \mathrm{~S}$ or $\mathrm{NH}_{3}$ neal the 5 bar level. In the second method, clouds are presumed a priori to occur where an assumed chemical component is predicted to condense. The models of Danielson (1975) are of this type and are essentially single $\mathrm{CH}_{4}$ cloud models. 
In step (D), great advances have been made by Macy (1979) and wallace (1980) through the use of laboratory spectra of $\mathrm{CH}$ (Fink et al, 1977); Giver, 1978) in computing solar heat deposition as a function of depth in the atmosphere. The heating (in terms of ergs/gm/sec) peaks just above the 1 bar level, but its influence on structure may extend well below the 10 bar level depending on the precise nature of the lower cloud (cf., Wallace, 1980) and the magnitude of the internal heat flow.

Danielson (1975) was the first to explore the influence of heat deposition on structure with the help of a highly parameterized heating function. He was able to show that deep radiative structures might be possible for Uranus but I believe that models are now mainly of historical interest for they are all too cold and fail to give a reasonable account of the microwave spectrum between 0.1 and $1 \mathrm{~cm}$.

Step (E), which is the heart of the modelling method, involves the calculation of thermal opacity. Now usually based on the work of Birnbaum, (1978) and numerical tests of the local buoyant stability of the atmosphere. The latter are dependent on the assumed composition through the specific heat of the atmospheric mixture. Both the assumed proportion of $\mathrm{CH}_{4}$ and the ability of $\mathrm{H}_{2}$ to convert between its ortho and para varieties is important in the calculation of specific heat. At the low temperatures in the Uranus visible atmosphere so called "equilibrium" $\mathrm{H}_{2}$ (in which transitions between ortho and para states are assumed unhindered) and "normal" $\mathrm{H}_{2}$ have very different specific heats, and the kind of $\mathrm{H}_{2}$ which is assumed leads to quite different stability conditions. Wallace (1986) has made a major point of including this problem in his calculations and carries three assumptions as to the state of $\mathrm{H}_{2}$ : (i) "normal" $\mathrm{H}_{2}$ in which the ortho-para ratio is effectively assumed to be that imposed at high temperature deep in the atmosphere, (iii) "equilibrium" $\mathrm{H}_{2}$ in which ortho-para conversion is unhindered by quantum mechanical restriction at any temperature, and (iii) "intermediate" $\mathrm{H}_{2}$, first postulated by Trafton (1967), in which the ortho-para ratio responds (presumably on a very long time scale) to conversion at the mean local temperature but does not respond on the short time scales of local convective processes in the atmosphere. Thus, "intermediate" hydrogen reflects a population of rotational states similar to "equilibrium" $\mathrm{H}_{2}$ but has a specific heat similar to "normal" hydrogen. I think that 2 n this application to uranus Wallace has convincingly shown that it is to this "intermediate" case that $\mathrm{H}_{2}$ prefers to conform in the atmosphere of the outer planets.

\section{(b) Wallace's (1980) Models}

The essential ingredients of the successful model, chosen to give the widest satisfaction to the complete set of observations, rather 
than precise agreement to any particular observation, are as follows:

(i) a Trafton (1976) type cloud and haze structure consisting of a diffuse, highly reflective, but optically thin haze of probably $\mathrm{CH}_{4}$, near 1 bar which is separated from a thick (in visual wavelengths) cloud base near the 5 bar level. This lower cloud takes on an albedo to sunlight of 0.98 (and is probably $\mathrm{H}_{2} \mathrm{~S}$ or $\mathrm{NH}_{3}$ ).

(ii) a ubiquitous, lightly absorbing haze (photochemical "detrius"?)

(iii) "intermediate" hydrogen

(iv) a mixing ratio for methane of 0.03 relative to $\mathrm{H}_{2}$.

The best models (shown in Fig. 17 of Wallace, (1980) represent, in my view, the best approximation to the mean atmospheric structure on Uranus between the 0.1 and 30 bar level available at this time. Below 30 bars, the structure is uncertain and depends partly on what is assumed about the magnitude of the internal heat flux and partly on the optical properties of the base cloud at 5 bars. Above 0.1 bars, the structure is similarly uncertain depending on the precise nature and mechanisms of any tropopausal "cold trap", on the particular nature of the photochemistry, and on whatever energy transport and disipation mechanisms exist in that region.

Wallace's comparison of his models with observational data is instructive: The models predict Bond albedos and effective temperature and since all of them give an adequate account of these quantities (Bond albedo between 0.315 and 0.361 ; effective temperatures between 56.9 and $60^{\circ} \mathrm{K}$ ) it turns out that these parameters do not help discriminate between the models but merely represent an overall check of their validity.

The predicted IR spectrum between 10 and $300 \mu$ turns out to be a poor discriminator between models, primarily because of the very poor quality and the inconsistency in the data set.

It is the microwave spectrum between $1-10 \mathrm{~mm}$ and in the visual and near infrared spectrum that the most leverage to discriminate between the various models is found. The mm observations rule out all "equilibrium" $\mathrm{H}_{2}$ models and those "intermediate" $\mathrm{H}_{2}$ models with too much $\mathrm{CH}_{4}$. The $\mathrm{CH}_{4} / \mathrm{H}_{2}$ ratio is restricted to less than ${ }^{2} 0.1$. 
In the visible and near infrared reflection spectrum, the $\mathrm{CH}_{4}$ "blue" bands put a lower limit on the amount of methane and require 4 $\mathrm{CH}_{4} / \mathrm{H}_{2}>0.01$; while a combination of the $\mathrm{H}_{2}$ spectrum and the strong $\mathrm{CH}$ bands ${ }^{2}$ near. $8 \mu$ set the character of the cloud structure as explained pre 4 viously.

The models place little constraint on the internal heat flux although the comparison with the microwave spectrum seems to prefer the models with higher internal heat flow.

However, this is barely a significant result, if at all.

\section{Unsolved Problems, Speculation, and Future Observations}

The outstanding contemporary problems in structure and composition that I believe need to be addressed in the immediate future are as follows :

(i) Why does Neptune have so much more cloud activity in the upper troposphere then Uranus?

(ii) What seasonal variations occur in the Uranus atmosphere; particularly near the tropopausal minimum?

(iii) What is the explanation of the shape and variability of Uranus' microwave spectrum between 1 and $21 \mathrm{~cm}$ ?

(iv) What is the explanation of the apparently low atmospheric deuterium and nitrogen abundances?

The first three problems are probably related and will require high quality observations in the 5 - $30 \mu$ region over an extended time base together with more occultations and observations particularly from voyager. Problems (iii) and (iv) may also be interrelated. The microwave spectrum at wavelengths longer than $2 \mathrm{~cm}$ seems to demand either a local or overall depletion of $\mathrm{NH}_{3}$ by a factor of 100 relative to solar abundance depending on the particular hypothesis that is made regarding the deep atmospheric structure. Excluding what seems to be clearly unacceptable hypotheses involving ionospheric emission and magnetospheric emission, the following ideas have been proposed as possible explanations of the shape of the microwave spectrum:

(a) depletion of $\mathrm{NH}_{3}$ coupled with a deep, roughly isothermal region below $30^{3}$ bars ( $250^{\circ} \mathrm{K}$ ) 
(b) a "surface" near the $280^{\circ} \mathrm{K}$ level (plus a depletion of $\mathrm{NH}_{3}$ ).

(c) chemical removal of $\mathrm{NH}_{3}$ at the $150^{\circ} \mathrm{K}$ level by a super abundance of $\mathrm{H}_{2} \mathrm{~S}$.

Idea (a) seems unacceptable because it does not provide a basis for an explanation of Neptune's microwave spectrum that has similar properties (Morrison and Cruikshank, 1973). Idea (b) might be acceptable if the equation of state of cosmic ices can accommodate an ice ball as large as Uranus and still meet the constraints of the mean density, as well as accommodate Neptune's large internal heat flow. In the context of this idea, the low $\mathrm{N}$ and $\mathrm{D}$ abundances would be explained by keeping these atoms largely trapped within the "ice ball." Idea (c) may work for both planets but leaves the deuterium problem unresolved. This idea also presents an untested hypothesis regarding the sulphur content of the atmosphere. Observations of HD on Neptune are needed as are more sensitive attempts to detect $\mathrm{H}_{2} \mathrm{~S}$. 
REFERENCES

Belton, M. J. S., McElroy, M. B., and Price, M. J. (1971)

"The Atmosphere of Uranus," Astrophys. Journ., Vol. 164, 191 - 209.

Belton, M. J. S., and Spinrad, H. (1973) " $\mathrm{H}$ Pressure-Induced Lines in the Spectra of the Major Planets," Astrophy. Journ., Vol. $185,363-372$.

Belton, M. J. S., Wallace, L., and Price, M. J. (1973) "Observation of the Raman Effect in the Spectrum of Uranus," Astrophys. Journ. (Letters), Vol. 184, L143 - L146.

Birnbaum, G. (1978) "Far Infrared Absorption in $\mathrm{H}_{2}$ and $\mathrm{H}_{2}-\mathrm{He}$ Mixtures," J. Quant. Spectros. Radiat. Transter, Vol. 19, $51-62$.

Brault, J. W., and Smith, W. H. (1980) "Determination of the $\mathrm{H}_{2}$ 4-OS(1) Quadrupole Line Strength and Pressure Shift," Astrophys. Journ. (Letters), Vol. 235, L177 - L178.

Briggs, F. H. (1973) "Observations of Uranus and Saturn by a New Method of Radio Interferometry of Faint Moving Sources," Astrophys. Journ., Vol. 182, 999 - 1011.

Capone, L. A., Whitten, R. C., Prasad, S. S., and Dubach, J. (1977) "The Ionosphere of Saturn, Uranus, and Neptune," Astrophys. Journ., Vol. 215, 977 - 983.

Courtin, R., Gautier, D., and Lacombe, A. (1978) "On the Thermal Structure of Uranus from Infrared Measurements," Astron. and Astrophys., Vol. 63, 97 - 101.

Dalgarno, A., and Allison, A. C. (1969) "Rotation-Vibration Quadrupole Matrix Elements and Quadrupole Absorption Coefficients of the Ground Electronic States of $\mathrm{H}_{2}$, HD and $\mathrm{D}_{2}, " \mathrm{~J}$. Atmos. Sci., Vol. 26, 946 - 951.

Danielson, R. E., Tomasko, M. G., and Savage, B. D. (1972)

"High Resolution Imagery of Uranus Obtained by Stratoscope II," Astrophys. Journ., Vol. 178, 887 - 900. 
Danielson, R. E. (1974) "The Visible Spectrum of Uranus," Astrophys. Journ. (Letters), Vol. 192, L107 - Lllo.

Danielson, R. E. (1975) "The Structure of the Atmosphere of Uranus," in "The Atmosphere of Uranus," Ed. D. M. Hunten, NASA Ames Workshop Proceedings.

Danielson, R. E., Cochran, W. D., Wannier, P. G., and Light, E. S. (1977) "A Saturation Model of the Atmosphere of Uranus" Icarus, Vol. 31, 97 - 109.

Dunham, D. W. (1971) "The Motions of the Satellites of Uranus," Ph. D. Thesis, Yale University.

Dunham, E., Elliot, J. L., and Gierasch, P. J. (1980) "The Upper Atmosphere of Uranus: Mean Temperature and Temperature

- Variations," Astrophys. Journ., Vol. 235, $274-284$.

Fink, U., Benner, D. C., and Dick, K. A. (1977) "Band Model Analysis of Laboratory Methane Absorption Spectra from 4500 to $10,500 \AA$," J. Quant. Spectrosc. Radiat. Transfer., Vol. $18,447-457$.

Fink, U., and Larson, H. P. (1979) "The Infrared Spectra of Uranus, Neptune and Titan from 0.8 to 2.5 Microns," Astrophys. Journ., Vol. 233, 1021 - 1040.

Gillett, F. C., and Rieke, G. H. (1977) "5 - 20 Micron Observations of Uranus and Neptune," Astrophys. Journ. (Letters), Vol. 218, L141 - L144.

Giver, L. P. (1978) "Intensity Measurements of the $\mathrm{CH}$ Bands in the Region $4350 \AA$ to $10,600 \AA, " \mathrm{~J}$. Quant. Spectrosc. Radiat. Transfer, Vol. 19, $311-322$.

Gulkis, S. (1975) "Microwave Radiometry and Implications," in "The Atmosphere of Uranus," Ed. D. M. Hunten, NASA Ames Workshop Proceedings.

Gulkis, S., Janssen, M. A., and Olsen, E. T. (1978) "Evidence for the Depletion of Ammonia in the Uranus Atmosphere," Icarus, Vol. 34, $10-19$

Hubbard, w. B., and MacFarlane, J. J. (1980) "Structure and Evolution of Uranus and Neptune," J. Geophys. Res., Vol. 85, 22 
Hubbard, W. B., and MacFarlane, J.J. (1981) "Theoretical Predictions of Deuterium Abundances in the Jovian Planets," preprint.

Hunten, D. M. (1981) "Deep Atmospheric Temperatures for Uranus and Neptune," Private communication, unpublished.

Joyce, R. R., Pilcher, C. B., Cruikshank, D. P., and Morrison, D. (1977) "Evidence for Weather on Neptune I," Astrophys. Journ., Vol. 214, $657-662$.

Klein, M. J., and Turanago, J. A. (1978) "Evidence of an Increase in the Microwave Brightness Temperature of Uranus," Astrophys. Journ. (Letters), Vol. 224, L31 - L34.

Klepczynski, W. J., Seidelmann, P. K., and Duncombe, R. L. (1970) "The Masses of Saturn and Uranus," Astron. Journ., Vol. 75, 739 - 742 .

Lutz, B. L., Owen, Tobias, and Cess, R. D. (1976) "Laboratory Band Strengths of Methane and their Application to the Atmospheres of Jupiter, Saturn, Uranus, Neptune, and Titon," Astrophys. Journ., Vol. 203, 541 - 551.

Macy, W. W., and Sinton, W. M. (1977) "Detection of Methane and Ethane Emission on Neptune but not on Uranus," Astrophys. Journ. (Letters), Vol. 218, L79 - L81.

Macy, W. W. (1979) "On the Clouds of Uranus," Icarus, Vol. 40, $213-222$.

Macy, w. W., Sinton, W. M., and Beichman, C. A. (1980) "FiveMicrometer Measurements of Urarius and Neptune," Icarus, vol. $42,68-70$.

McElroy, M. B. (1973) "The Ionosphere of the Major Planets," Space Science Rev., Vol. 14, 460 - 473.

Michalsky, J. J., and Stokes, R. A. (1977) "Wholedisk Polarization Measurements of Uranus at Visible Wavelengths," Astrophys. Journ. (Letters), Vol. 213, L135 - L137.

Morrison, D., and Cruikshank, D. P. (1973) "Temperature of Uranus and Neptune at 2.4 Microns," Astrophys. Journ., Vo1. 179, 329 - 331. 
Pilcher, C. B., Morgan, J. S., Macy, W. W., and Kunkle, T. D. (1979) "Methane Band Limb-Brightening on Uranus," Icarus, Vol. 39, $54-64$.

Price, M. J., and Franz, O. G. (1979) "Uranus: Disk Structure within the $7300 \AA$ Methane Band," Icarus, Vol. 39, $459-472$.

Prinn, R. G., and Lewis, J. S. (1973) "Uranus Atmosphere: Structuxe and Composition," Astrophys. Journ., Vol. 179, 333 342 .

Savage, B. D., Cochran, W. D., and Wesselius, P. R. (1980) "Ultraviolet Albedos of Uranus and Neptune," Astrophys. Journ., Vol. 237, $627-632$.

Silvaggio, P. M. (1977) "Experimental Determination of Molecular Absorption Coefficients for Methane and Ammonia at Low Temperatures and Model Atmospheres for the Major Planets," Ph. D. Thesis, Cornell University.

Sinton, W. M. (1972) "Limb and Polar Brightening of Uranus at 8870§," Astrophys, Journ. (Letters), Vol. 176, L131 - L133.

Smith, W. H., Macy, W. W., and Pilcher, C. B. (1980) "Measurements of the $\mathrm{H}_{3} 4-0$ Quadrupole Bands of Uranus and Neptune," Icarus, vol. $43,153-160$.

Stier, M. T., Traub, W. A., Fozio, G. G., Wright, E. L., and Low, F. J. (1978) "For Infrared Observations of Uranus, Neptune, and Ceres," Astrophys. Journ., Vol. 226, $347-349$.

Trafton, L. (1967) "Model Atmospheres of the Major Planets," Astrophys. Journ., Vol. 147, 765 - 781.

Trafton, L. (1972) "On the Methane Opacity for Uranus and Neptune," Astrophys. Journ. (Letters), Vol. 172, L117 - Ll20.

Trafton, L. (1976) "The Aerosol Distribution in Uranus' Atmosphere: Interpretation of the Hydrogen Spectrum," Astrophys, Journ., Vol. 207, 1007 - 1024.

Trafton, L. and Ramsay, D. A. (1980) "The D/H Ratio in the Atmosphere of Uranus: Detection of the $R_{5}(1)$ Line of HD," Icarus, Vol. $41,423-429$. 
Trauger, J. T., Roesler, F. L., and Mickelson, M. (1977) "The D/H Ratio on Jupiter, Saturn, and Uranus Based on New HD and $\mathrm{H}_{2}$ Data," Bull. Amer. Astron. Soc., Vol. 9, 516.

Wallace, L. (1975) "On the Thermal Structure of Uranus," Icarus, Vol. $25,538-544$.

Wallace, L., (1980) "The Structure of the Uranus Atmosphere," Icarus, Vol. 43, 231 - 259.

'ounkin, R. L. (1970) "Spectrophotometry of the Moon, Mars and Uranus," Dissertation, University of California, Los Angeles. 